\title{
Growth hormone therapy and effect on ovarian function and morphology in short prepubertal SGA girls
}

Jeanette Tinggaard1, Rikke Beck Jensen ${ }^{1}$, Karin Sundberg², Katharina M. Main ${ }^{1}$, Niels Birkebaek ${ }^{3}$, Peter Christiansen ${ }^{1}$, Annie Ellermann ${ }^{4}$, Kirsten Holm $^{5}$, Eva Mosfeldt-Jeppesen ${ }^{6}$, Nina Saurbrey ${ }^{6}$, Britta Kremke ${ }^{7}$, Pawel Marcinski ${ }^{8}$, Rune Weis Naeraa ${ }^{3}$, Carsten Pedersen ${ }^{9}$, Brigitte Scherling ${ }^{10}$, Ebbe Thisted ${ }^{11}$, the NESGAS group ${ }^{*}$, Anders Juul ${ }^{1}$

1 University Department of Growth and Reproduction, Rigshospitalet, Copenhagen, Denmark, 2 Department of Fetal Medicine, Rigshospitalet, Copenhagen, Denmark, 3 Department of Pediatrics, Aarhus University Hospital Skejby, Denmark, 4 Department of Pediatrics, Holbæk sygehus, Denmark, 5 Department of Pediatrics, Hillerød sygehus, Denmark, 6 Department of Pediatrics, Glostrup Hospital, Denmark, 7 Department of Pediatrics, Randers sygehus, Denmark, 8 Department of Pediatrics, Vendsyssel sygehus Hjørring, Denmark, 9 Department of Pediatrics, Kolding sygehus, Denmark, 10 Department of Pediatrics, Roskilde sygehus, Denmark, 11 Department of Pediatrics, Sønderborg Sygehus, Denmark.

Disclosure statement: This study was supported by Novo Nordisk A/S

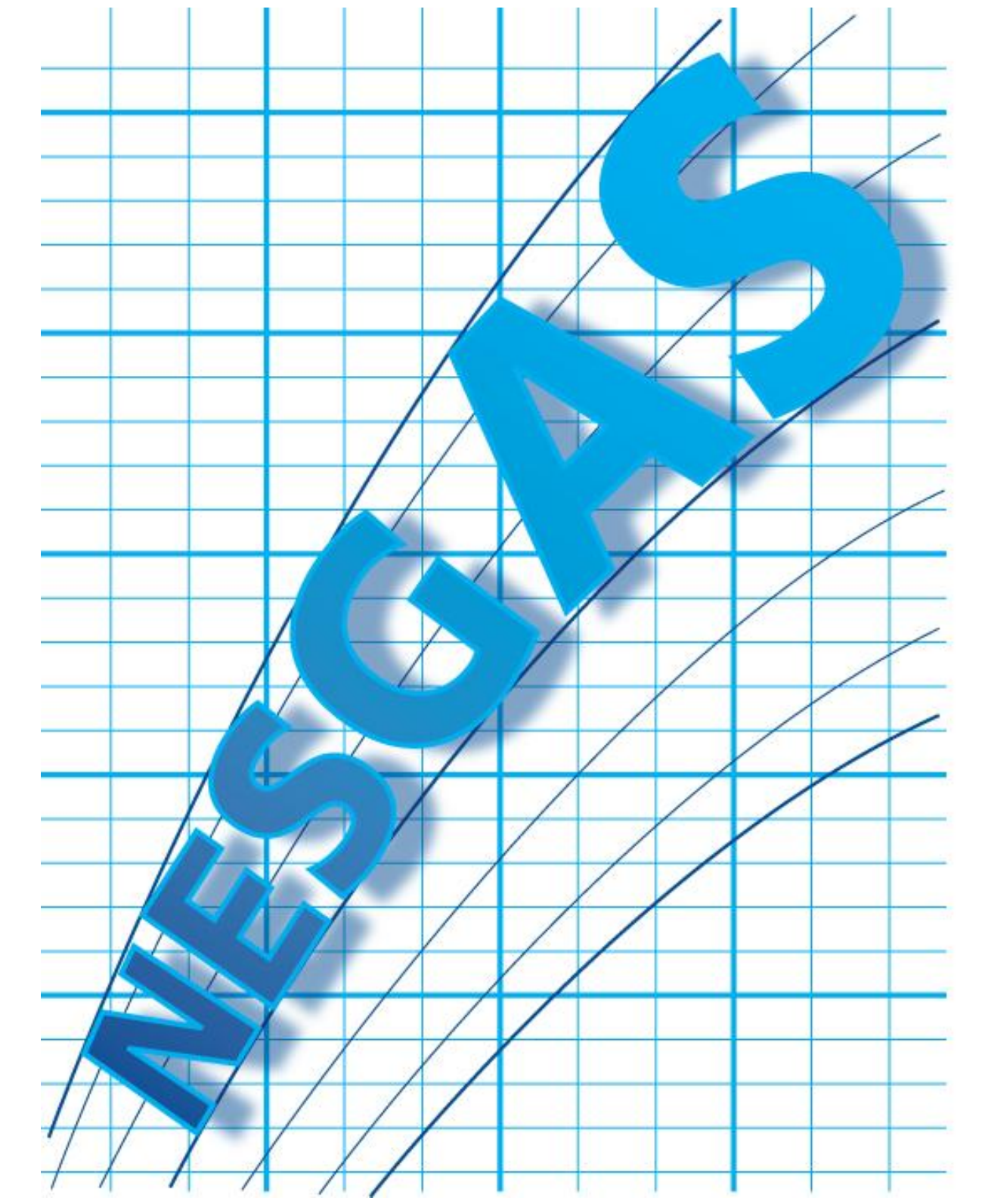

\section{Background}

Girls born small for gestational age (SGA) are at risk of early pubertal maturation and development of PCOS. Growth Hormone $(\mathrm{GH})$ receptors are present in ovaries, and $\mathrm{GH}$ may have a physiological role for ovarian function, but little is known about the effect of GH therapy on ovarian function in short girls born SGA. The objective of this study was to evaluate ovarian morphology and function during GH therapy.

\section{Methods}

Clinical characteristics, reproductive hormones and ultrasonographic examination of the internal genitalia were determined in 18 prepubertal girls during the first three years of GH therapy in a Danish sub-study of the North European SGA study (NESGAS); a multinational, randomised, longitudinal study of $\mathrm{GH}$ therapy in short prepubertal children born SGA.

\section{Results}

Median age at baseline was 4.9 years (4.57.2) (Table 1). Bone age advanced significantly during three years of treatment $(P=0.007)$, but did not exceed chronological age. Uterine and ovarian volume increased significantly $\quad(P=0.033$ and $P=0.005$, respectively), but remained low within the normal reference ranges (Figure 1, left panel). Ovarian follicles became visible in $69 \%$ of girls compared to $28 \%$ of girls before $\mathrm{GH}$ therapy. No significant changes in follicular number or size were found. Precocious puberty was observed in one girl and another girl showed signs of a multicystic ovary verified by ultrasound. Anti-Müllerian Hormone tended to cluster in the lower part of the reference range, although a significant increase during three years of $\mathrm{GH}$-treatment was observed $(P=0.028)$ (figure 1, right panel). SHBG decreased during the first year of $\mathrm{GH}$ therapy $(\mathrm{P}<0.001)$ and remained low, while an increase in $\Delta 4$-androstenedione and DHEAS was found. Inhibin $B$ increased significantly, but no significant changes in FSH, LH, estradiol or inhibin A were found.
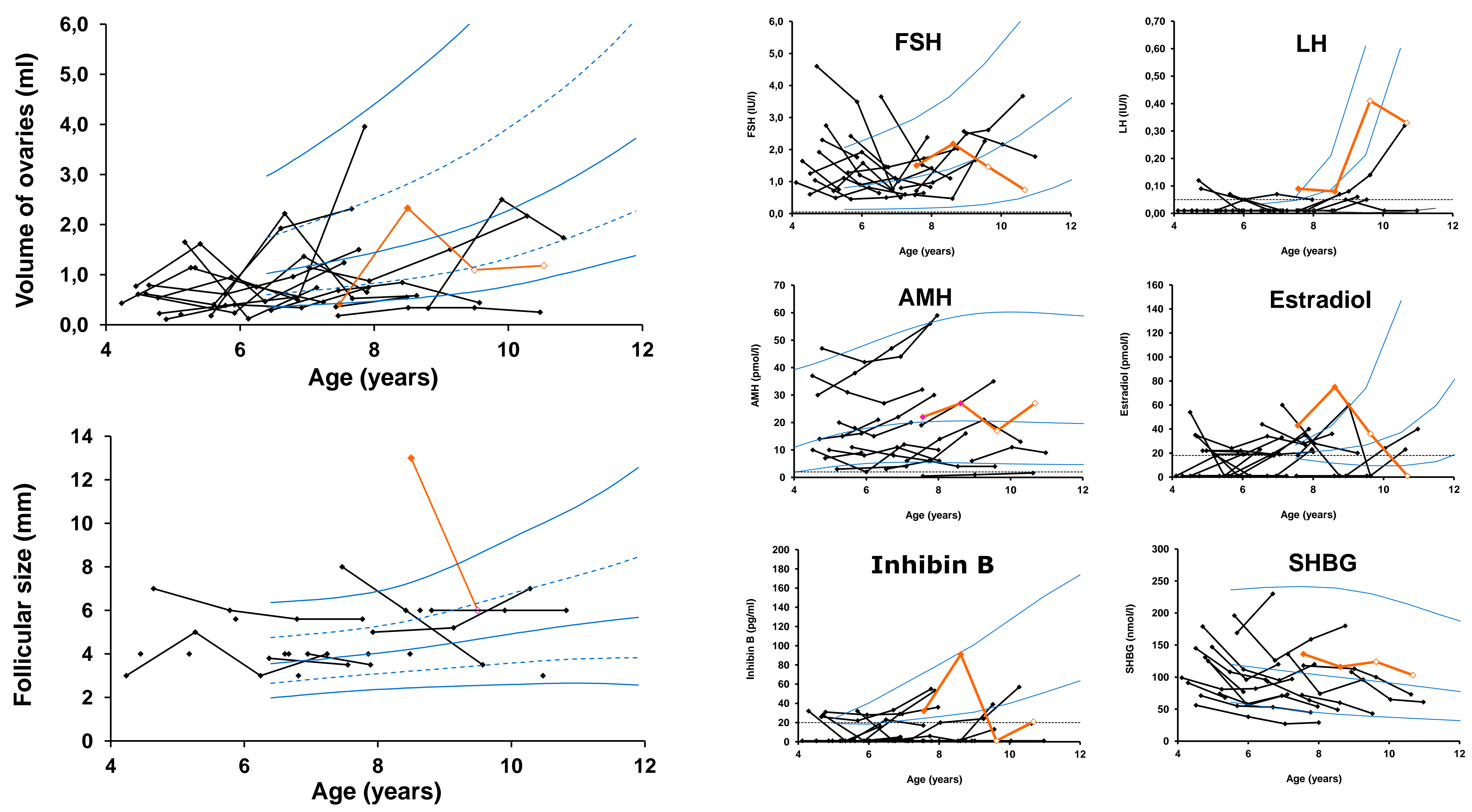

Figure 1, left panel; Volume of ovaries (upper figure) and follicular size (lower figure) according to age during GH therapy in 18 short prepubertal SGA girls. Figure 1, right panel: Circulating serum levels of FSH, LH, AMH, Estradiol, Inhibin B and SHBG according to age during GH therapy in 18 short prepubertal SGA girls. Solid blue lines represent mean $+/-2$ SD and dotted blue lines represent $+/-1$ SD. One girl who presented with precocious puberty is highlighted in colour with filled squares before and blank squares after treatment with a GnRH agonist.

\begin{tabular}{|c|c|c|c|c|}
\hline & $\begin{array}{l}\text { Baseline } \\
(n=18)\end{array}$ & $\begin{array}{l}1 \text { year } \\
(n=17)\end{array}$ & $\begin{array}{l}2 \text { year } \\
(n=17)\end{array}$ & $\begin{array}{l}3 \text { year } \\
(n=14)\end{array}$ \\
\hline $\begin{array}{l}\text { Chronological } \\
\text { Age (yr) }\end{array}$ & $\begin{array}{l}4.91 \\
(4.51-7.22)\end{array}$ & $\begin{array}{l}5.95 \\
(5.58-8.32)\end{array}$ & $\begin{array}{l}6.95 \\
(6.59-9.39)\end{array}$ & $\begin{array}{l}7.98 \\
(7.50-9.74)\end{array}$ \\
\hline $\begin{array}{l}\text { Bone age } \\
\text { (BoneXpert) }\end{array}$ & $\begin{array}{l}3.27 \\
(2.09-5.74)\end{array}$ & $\begin{array}{l}4.49 \\
(3.23-6.81)\end{array}$ & $\begin{array}{l}6.56 \\
(5.41-7.87)\end{array}$ & $\begin{array}{l}7.20 \\
(6.22-8.71)\end{array}$ \\
\hline BA-CA & $\begin{array}{l}-1.64 \\
(-2.53 \text { to }-1.13)\end{array}$ & $\begin{array}{l}-1.59 \\
(-2.17 \text { to }-1.22)\end{array}$ & $\begin{array}{l}-1.05 \\
(-1.78 \text { to }-0.23)^{*}\end{array}$ & $\begin{array}{l}-1.14 \\
(-1.71 \text { to } 0.42)^{\#}\end{array}$ \\
\hline Weight (SDS) & $\begin{array}{l}-2.88 \\
(-3.24 \text { to }-1.67)\end{array}$ & $\begin{array}{l}-1.72 \\
(-2.50 \text { to }-0.56)^{*}\end{array}$ & $\begin{array}{l}-1.38 \\
(-2.30 \text { to } 0.15)^{*}\end{array}$ & $\begin{array}{l}-1.57 \\
(-2.08 \text { to } 0.10)^{* \#}\end{array}$ \\
\hline Height (SDS) & $\begin{array}{l}-2.95 \\
(-3.47 \text { to }-2.62)\end{array}$ & $\begin{array}{l}-1.97 \\
(-2.69 \text { to }-1.47)^{*}\end{array}$ & $\begin{array}{l}-1.56 \\
(-2.39 \text { to }-0.98)^{*}\end{array}$ & $\begin{array}{l}-1.41 \\
(-2.00 \text { to } 0.35)^{\star \#}\end{array}$ \\
\hline BMI (SDS) & $\begin{array}{l}-0.60 \\
(-1.71 \text { to }-0.25)\end{array}$ & $\begin{array}{l}-0.71 \\
(-1.83 \text { to }-0.61)\end{array}$ & $\begin{array}{l}-0.43 \\
(-1.81 \text { to } 0.69)\end{array}$ & $\begin{array}{l}-0.79 \\
(-1.93 \text { to }-0.64)\end{array}$ \\
\hline Tanner stage I/II & $18 / 0$ & $16 / 1$ & $15 / 2$ & $13 / 1$ \\
\hline $\begin{array}{l}\text { Uterine volume } \\
\text { (ml) }\end{array}$ & $\begin{array}{l}1.05 \\
(0.68-1.56)\end{array}$ & $\begin{array}{l}1.22 \\
(0.88-2.32)^{*}\end{array}$ & $\begin{array}{l}1.50 \\
(1.04-2.20)\end{array}$ & $\begin{array}{l}1.72 \\
(0.88-2.45)^{\#}\end{array}$ \\
\hline $\begin{array}{l}\text { Ovarian volume } \\
\text { (ml) }\end{array}$ & $\begin{array}{l}0.43 \\
(0.31-0.69)\end{array}$ & $\begin{array}{l}0.69 \\
(0.39-1.63)\end{array}$ & $\begin{array}{l}0.76 \\
(0.47-1.30)\end{array}$ & $\begin{array}{l}0.90 \\
(0.62-1,84)^{\#}\end{array}$ \\
\hline $\begin{array}{l}\text { Largest follicle } \\
(\mathrm{mm})\end{array}$ & $\begin{array}{l}5.0 \\
(3.3-6.8)\end{array}$ & $\begin{array}{l}5.3 \\
(4.0-6.5)\end{array}$ & $\begin{array}{l}4.6 \\
(3.6-6.0)\end{array}$ & $\begin{array}{l}4.0 \\
(3.5-5.9)\end{array}$ \\
\hline FSH (IU/I) & $\begin{array}{l}1.50 \\
(0.89-2.50)\end{array}$ & $\begin{array}{l}1.33 \\
(0.73-2.04)\end{array}$ & $\begin{array}{l}1.11 \\
(0.75-1.70)\end{array}$ & $\begin{array}{l}0.98 \\
(0.63-1.64)\end{array}$ \\
\hline LH (IU/I) & $\begin{array}{l}0.01 \\
(0.01-0.06)\end{array}$ & $\begin{array}{l}0.01 \\
(0.01-0.01)\end{array}$ & $\begin{array}{l}0.01 \\
(0.01-0.05)^{*}\end{array}$ & $\begin{array}{l}0,05 \\
(0.01-0.06)\end{array}$ \\
\hline AMH (pmol/l) & $11(6-22)$ & $14(8-27)$ & $21(11-35)$ & $20(8-31) \#$ \\
\hline Estradiol (pmol/l) & $18(1-39)$ & $19(1-26)$ & $20(10-36)$ & $20(14-26)$ \\
\hline SHBG (nmol/l) & $125(92-146)$ & $74(62-110)^{*}$ & $84(55-98)$ & $80(52-99) \#$ \\
\hline DHEAS (mmol/l) & $\begin{array}{l}0.47 \\
(0.01-0.56)\end{array}$ & $\begin{array}{l}0.51 \\
(0.01-1.23)^{*}\end{array}$ & $\begin{array}{l}0.79( \\
0.71-2.05)^{*}\end{array}$ & $\begin{array}{l}1.36 \\
(1.26-1.61)^{* \#}\end{array}$ \\
\hline $\begin{array}{l}\text { Androstenedione } \\
\text { (mmol/l) }\end{array}$ & $\begin{array}{l}0.01 \\
(0.01-0.01)\end{array}$ & $\begin{array}{l}0.01 \\
(0.01-1.87)^{*}\end{array}$ & $\begin{array}{l}0.01 \\
(0.01-1.50)\end{array}$ & $\begin{array}{l}1.19 \\
(0.01-1.93)^{\#}\end{array}$ \\
\hline $\begin{array}{l}\text { Testosterone } \\
\text { (nmol/l) }\end{array}$ & $\begin{array}{l}0.01 \\
(0.01-0.01)\end{array}$ & $\begin{array}{l}0.01 \\
(0.01-0.01)\end{array}$ & $\begin{array}{l}0.01 \\
(0.01-0.01)\end{array}$ & $\begin{array}{l}0.01 \\
(0.01-0.01)\end{array}$ \\
\hline
\end{tabular}

Table 1 Clinical characteristics and hormone analyses at baseline and after one, two and three years of GH treatment. Results are presented as medians (interquartile range). ${ }^{*} \mathrm{P}<0.05$ compared to previous year by Wilcoxon sign rank test, ${ }^{*} \mathrm{P}<0.05$ for values at three years compared to baseline.

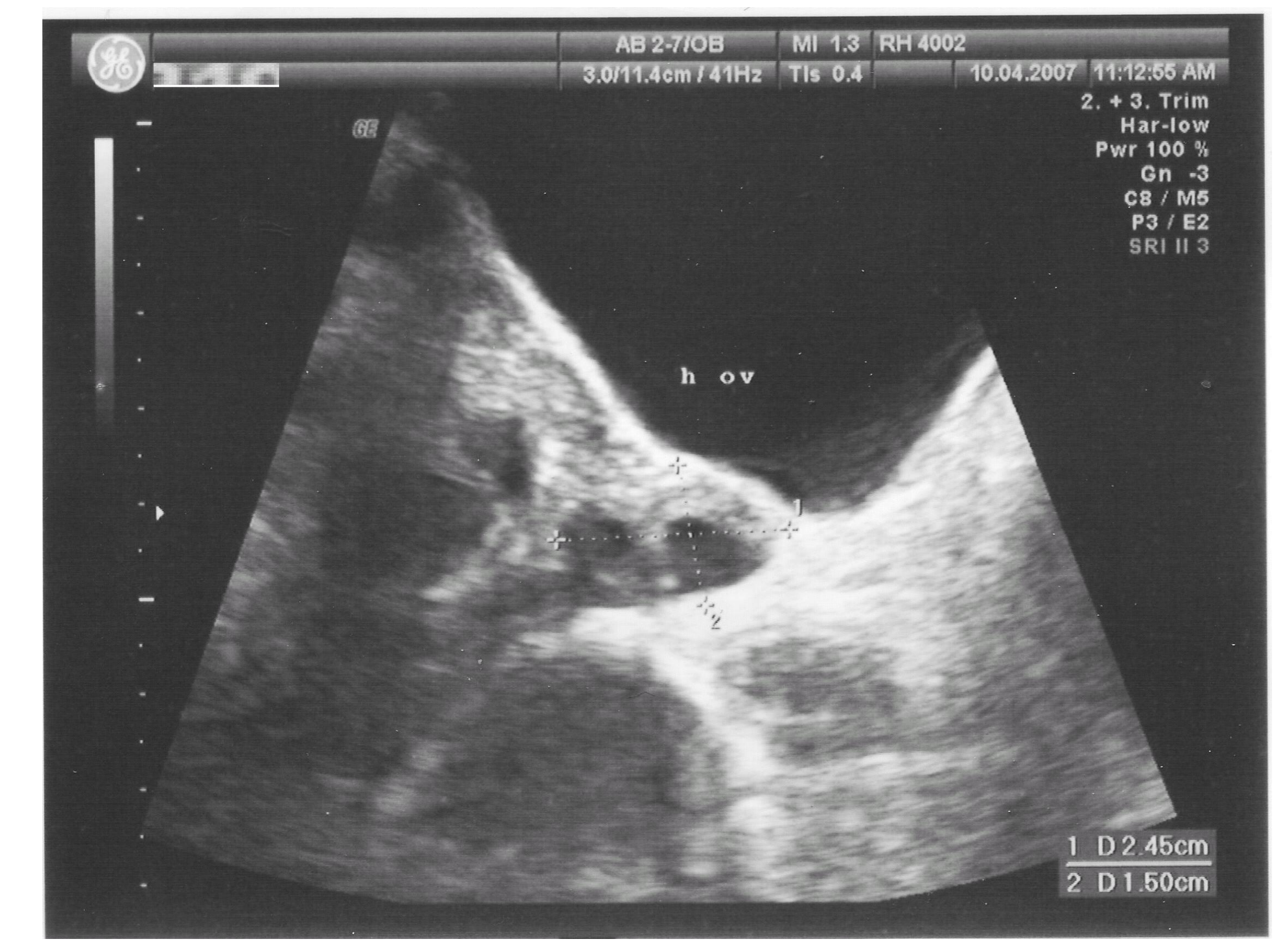

Figure 2. Ultrasonographic examination of the right ovary showing follicular activity

\section{- Conclusions}

GH therapy was associated with marked increase in height, and considered safe. Size and morphology of internal genitals increased proportionally to the increased body size during GH therapy. However, 2 of 18 girls developed precocious puberty (central) or multicystic ovary, respectively. It remains uncertain whether or not these findings were associated with the concomitant GH therapy, or independent phenomena.

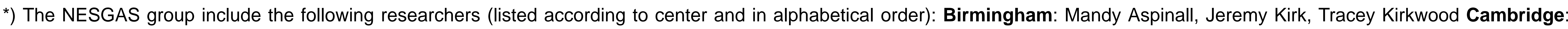

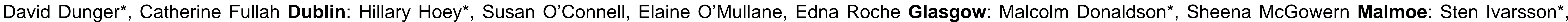
Helena Larsson, Carina Persson Lund: Maria Elfving, Lena Rollof Stockholm: Lo Neumeyer, Olle Söder*

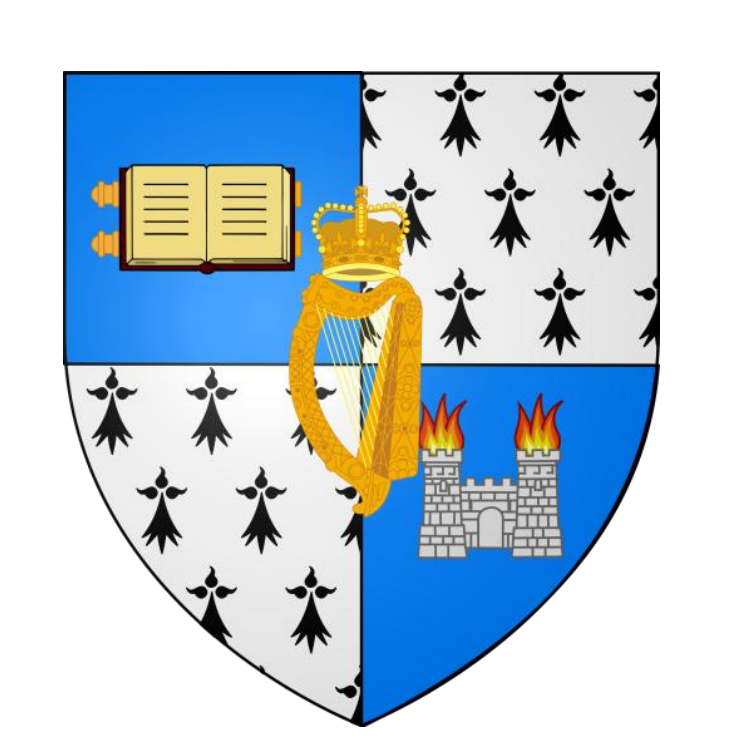

THE

UNIVERSITY

OF DUBLIN
圈圈 UNIVERSITY OF CAMBRIDGE

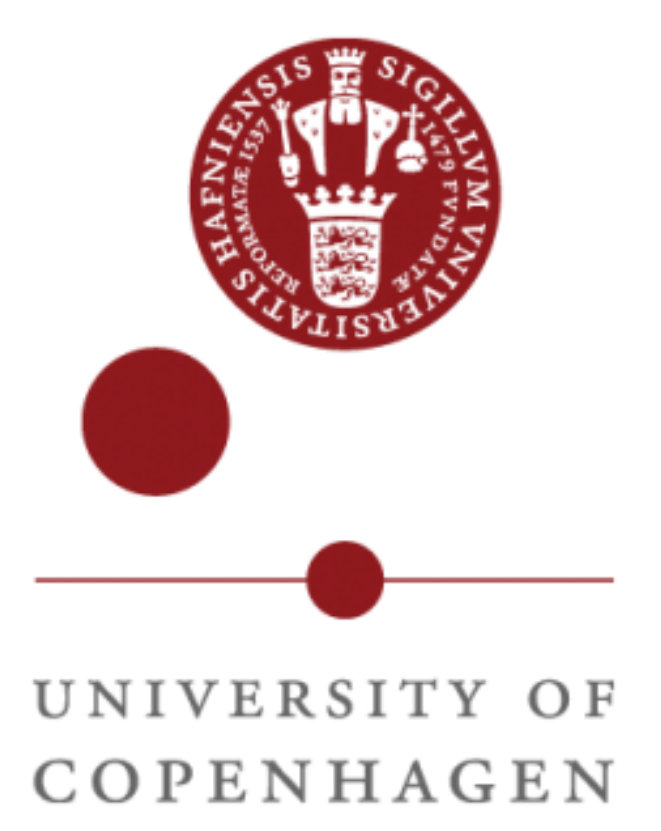

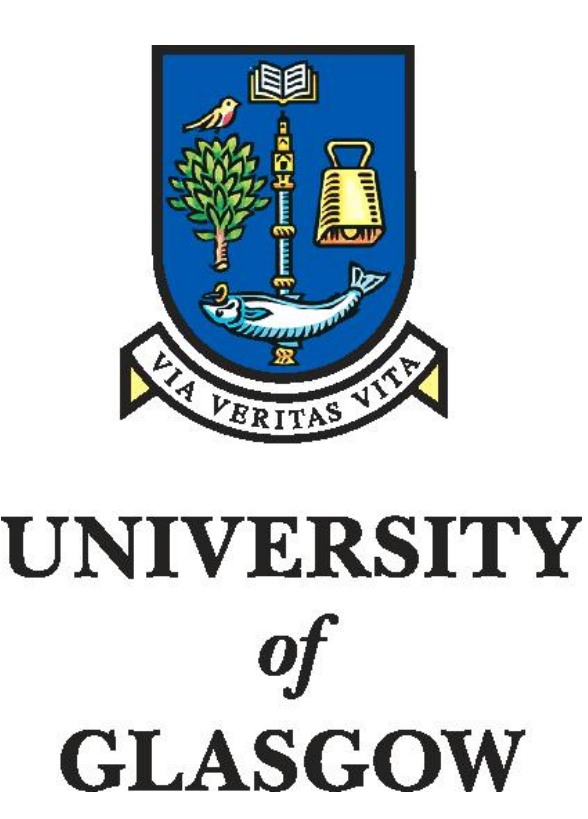

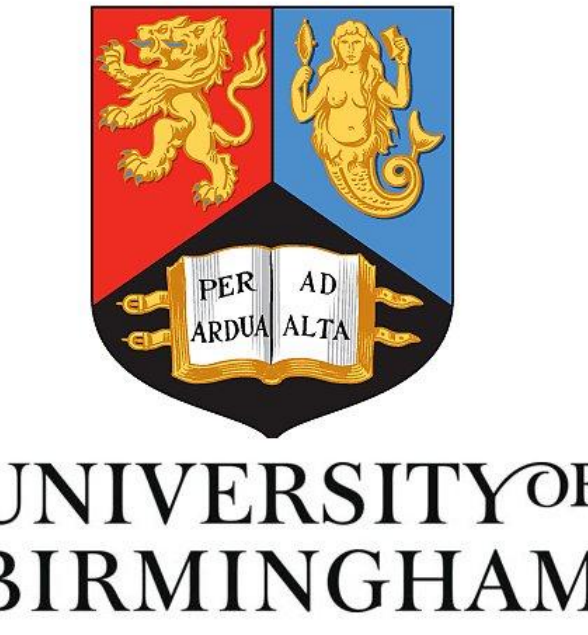

UNIVERSITYOF
BIRMINGHAM

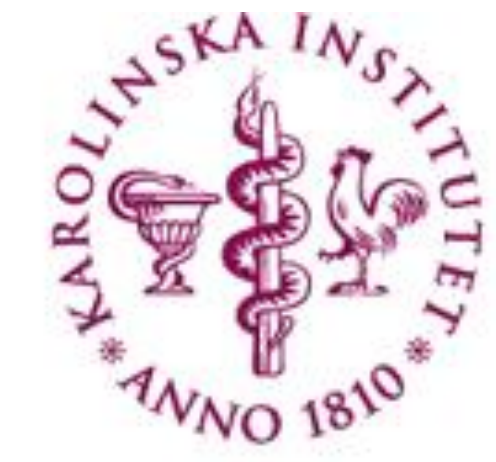

Karolinska

Institutet

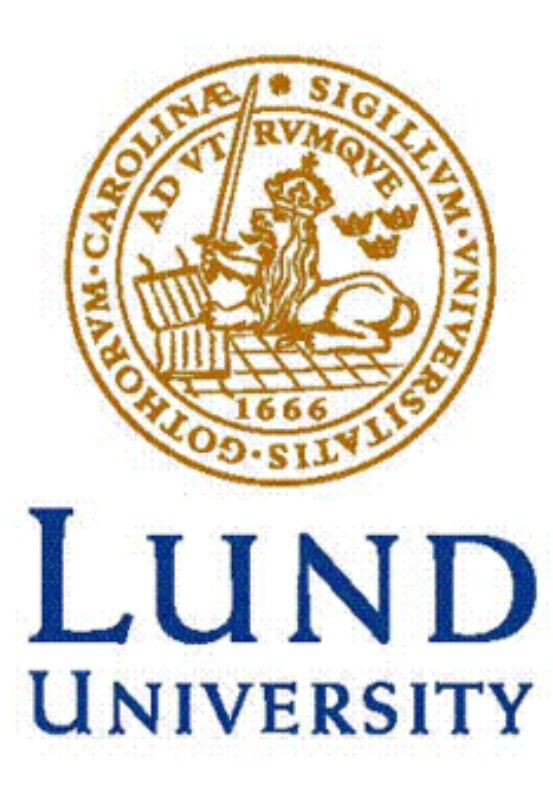

